\title{
Conformal Tensor Discontinuities in General Relativity*
}

\author{
L. C. SHEPLEY \\ University of Texas, Austin \\ Received April 8, 1968
}

\begin{abstract}
The postulate is made that across a given hypersurface $N$ the metric and its first derivatives are continuous. This postulate is used to derive conditions which must be satisfied by discontinuities in the Riemann tensor across $N$. These conditions imply that the conformal tensor jump is uniquely determined by the stress-energy tensor discontinuity if $N$ is non-null (and to within an additive term of type Null if $N$ is lightlike). Alternatively, $\left[C^{\alpha \beta}{ }_{\gamma \delta}\right]$ and $[R]$ determine

$$
\left[R_{\mu \nu}-\frac{1}{4} R g_{\mu \nu}\right]
$$

if $N$ is non-null. These relationships between the conformal tensor and stressenergy tensor jumps are given explicitly in terms of a three-dimensional complex representation of the antisymmetric tensors. Application of these results to perfectfluid discontinuities is made: $\left[C^{\alpha \beta} \beta_{\gamma \delta}\right]$ is of type $\mathrm{D}$ across a fluid-vacuum boundary and across an internal, non-null shock front. $\left[C^{\alpha \beta_{\gamma \delta}}\right]$ is of type I (non-degenerate) in general across fluid interfaces across which no matter flows, except for special cases.
\end{abstract}

\section{Introduction}

The satisfaction of conditions on the stress-energy tensor alone is necessary to ensure that a discontinuity across a hypersurface be acceptable [1], and much work has been done to study such conditions [2]. The rest of the Riemann tensor - the Conformal, or Weyl tensor therefore must have its discontinuities determined by the jump in $T^{\mu \nu}$. This fact has been known for interior-exterior junctions, and has appeared in the works of CockE [3] and of EsTABRooK and WAHLQUIST [4]. Here we will make explicit the algebraic form of the jump in $C^{\alpha \beta}{ }_{\gamma \delta}$ as given by a discontinuity in $T^{\mu \nu}$.

In Section II we will discuss the relationship between secondderivative metric jumps and Riemann tensor discontinuities. Section III will develop the language of three-dimensional complex vectors which will be used in the discussion of jumps in the Riemann tensor in Section

* The research reported herein was supported in part by the Atomic Energy Commission under contract number AT (11-1)-34, Project Agreement No. 125, while the author was at the University of California at Berkeley, and in part by National Science Foundation Grant GU-1598-University Science Development Program. 
IV. There it is shown that the discontinuity of $C^{\alpha \beta}{ }_{\gamma \delta}$ across $N$ is uniquely given by the jump in the stress-energy tensor $T^{\mu \nu}$, provided certain conditions are satisfied by [ $T^{\mu \nu}$ ] (in the case of $N$ non-null). Conversely, the jump in $\stackrel{R}{\mu \nu}_{\mu}$ is uniquely given by $\left[C^{\alpha \beta}{ }_{\gamma \delta}\right]$ and $[R]$ if the Weyl tensor jump satisfies certain conditions. Section $V$ discusses certain matterfilled models, and Section VI is a summary and conclusion.

Our sign conventions are that $g_{\mu \nu}$ is of signature $(-+++)$; the Riemann tensor is given by

$$
2 v_{\alpha ;[\beta \gamma]}=v_{\sigma} R_{\alpha \beta \gamma}^{\sigma}
$$

and the Ricci and reduced Ricci tensors are

$$
R_{\mu \nu}=R_{\mu \sigma v}^{\sigma} ; \stackrel{R}{\mu \nu}_{\mu \nu}=R_{\mu \nu}-\frac{1}{4} R g_{\mu \nu} .
$$

\section{Discontinuities in the Second Derivatives of the Metric}

We choose coordinates on either side of the hypersurface $N$ so that the jumps $\left[n_{\mu}\right],\left[g_{\mu \nu}\right],\left[g_{\mu \nu, \alpha}\right]$ all vanish ${ }^{1}$. That this choice can always be made has already been shown [1]. One consequence is that [5] $\left[g_{\mu v, \alpha \beta}\right]$ obeys

$$
\left[g_{\mu v, \alpha \beta}\right]=m_{\mu v} n_{\alpha} n_{\beta} .
$$

The jump $\left[g_{\mu \nu, \alpha \beta}\right]$ can be transformed away by a coordinate transformation if and only if it has the form

$$
\left[g_{\mu \nu, \alpha \beta}\right]=2 f_{(\mu} n_{\nu)} n_{\alpha} n_{\beta}, \quad \text { or } m_{\mu \nu}=2 f_{(\mu} n_{\nu)} .
$$

The proof takes two parts: First, note that if $\left[g_{\mu v, \alpha \beta}\right]$ has this form and if $N$ is given by the equation $x^{1}=0$, then the transformation

$$
\begin{aligned}
& \bar{x}^{\mu}=x^{\mu}-\frac{1}{6}\left(x^{1}\right)^{3} f^{\mu}\left(x^{1}\right) \text { for } x^{1}>0, \quad i \neq 1 \\
& \bar{x}^{\mu}=x^{\mu} \text { for } x^{1} \leqq 0
\end{aligned}
$$

does eliminate $\left[g_{\mu \nu, \alpha \beta}\right]$ in the $\bar{x}^{\mu}$ coordinates.

The second part of the proof uses the expression of $R_{\alpha \beta \gamma \delta}$ in terms of the derivatives of $g_{\mu \nu}$. If we write $m_{\mu \nu}$ as

$$
m_{\mu \nu}=2 f_{(\mu} n_{\nu)}+\bar{H}_{\mu \nu}
$$

then the $f_{(\mu} n_{v)}$ term does not contribute to $\left[R_{\alpha \beta \gamma \delta}\right]$ :

$$
\left[R_{\alpha \beta \gamma \delta}\right]=2 n_{[\alpha} \bar{H}_{\beta][\gamma} n_{\delta]} .
$$

In the case of $n_{\alpha}$ null, choose a null vector $p_{\alpha}$ such that $p^{\sigma} n_{\sigma}=1$, $p^{\alpha} p_{\sigma}=0$. The above expression for $\left[R_{\alpha \beta \gamma \delta}\right]$ is used to find

$$
\left[R_{\alpha \beta \gamma \delta}\right]\left(\delta_{\sigma}^{\alpha}-p^{\alpha} n_{\sigma}\right)\left(\delta^{\gamma}{ }_{\tau}-p^{\gamma} n_{\tau}\right)=-\frac{1}{2} \bar{H}_{\sigma \tau} n_{\beta} n_{\delta} .
$$

1 The notation $\left[n_{\mu}\right]=0$ indicates that $N$ has the same definition in terms of the coordinates on either side of $N$. The notation $[f]$ for a function defined in a neighborhood of $N$ indicates the difference in the limits of $f$ on the two sides of $N$. 
In the case of $n_{\alpha}$ non-null, $m_{\mu \nu}$ may be uniquely decomposed as

$$
m_{\mu \nu}=2 f_{(\mu} n_{\nu)}+\bar{H}_{u \nu}, \bar{H}_{\mu \sigma} n^{\sigma}=0 \text {. }
$$

In this case we find

$$
\bar{H}_{\beta \delta}=-2\left(n^{\sigma} n_{\sigma}\right)^{-1}\left[R_{\beta \delta}-\frac{1}{2} R\left(n^{\tau} n_{\tau}\right)^{-1} n_{\beta} n_{\delta}\right] .
$$

Eqs. (2.2) and (2.3) say that if and only if $\bar{H}_{\mu \nu}$ is non-zero there will be non-zero components in $\left[R_{\alpha \beta \gamma \delta}\right]$, whether $n_{\alpha}$ is null or non-null. Since $R_{\alpha \beta \gamma \delta}$ is a tensor, $\left[R_{\alpha \beta \gamma \delta}\right]$ is affected only by coordinate transformations whose first derivatives are discontinuous across $N$. But such a coordinate transformation cannot preserve the vanishing of $\left[g_{\mu \nu}\right]$ and $\left[g_{\mu \nu, \alpha}\right]$ in the case that $g_{\mu \nu}$ has no symmetries. (We will not go into the case of symmetric $g_{\mu \nu}$.) In general, therefore, $\left[g_{\mu \nu, \alpha \beta}\right]$ is removable if and only if $\left[R_{\alpha \beta \gamma \delta}\right]$ vanishes.

Eq. (2.3) anticipates some of the results of Section IV, since the right side is given by the jump in the stress-energy tensor. Note that in turn $\left[T_{\mu \nu}\right]$ is given by

so that

$$
\left[T_{\alpha \beta}\right]=-\frac{1}{2} n^{\sigma} n_{\sigma} \bar{H}_{\alpha \beta}-\frac{1}{2} \bar{H}_{\sigma}^{\sigma} n_{\alpha} n_{\beta}+\frac{1}{2} \bar{H}_{\sigma}^{\sigma} g_{\alpha \beta} n^{\tau} n_{\tau}
$$

$$
\left[T^{\alpha \sigma}\right] n_{\sigma}=0
$$

In Section IV, the result that $\left[T^{\mu \nu}\right]$ gives $\left[R_{\alpha \beta \gamma \delta}\right]$ will be spelled out in greater detail.

\section{Three-Dimensional Complex Vectors}

To discuss the algebra of $R_{\alpha \beta \gamma \delta}$, we will use for convenience a representation of the antisymmetric two-tensors given by three -component complex vectors $\gamma_{i}$ in a space $C^{3}$ [6]. On $C^{3}$ the metric $a_{i j}$ (usually taken as constant) is reducible by a coordinate transformation to $\delta_{i j}$, but we will not assume that $a_{i j}=\delta_{i j}$ to raise or lower three-dimensional indices. A complex vector $\gamma_{i}$ corresponds to a (complex) self-dual, antisymmetric two-tensor $\mathrm{F}^{+}{ }_{\mu \nu}$. The operation of taking a dual, denoted *, converts an antisymmetric tensor $F_{\mu \nu}$ to

$$
* F_{\mu \nu}=\frac{1}{2} \sqrt{g} \varepsilon_{\mu \nu \sigma \tau} F^{\sigma \tau}=\frac{1}{2} E_{\mu \nu \sigma \tau} F^{\sigma \tau}
$$

$\left(\varepsilon_{\mu \nu \sigma \tau}\right.$ is defined so that $\left.\varepsilon_{0123}=1\right)$. Note that $E_{\mu \nu \sigma \tau}$ is a pure imaginary tensor. Thus the * applied twice cancels itself, and a self-dual tensor is necessarily complex. The three independent self-dual tensors $\gamma_{i}^{\alpha \beta}$ 22 Commun. math. Phys., Vol. 9 
$(i=1,2,3)$ are chosen so that ${ }^{2}$

$$
\gamma_{i}{ }^{\mu \nu} \gamma_{j \mu \nu}=-4 a_{i j}
$$

Thus a self-dual tensor $F^{+}{ }_{\mu \nu}$ corresponds to the vector $f_{i}$ by:

$$
f_{i}=-\frac{1}{4} \gamma_{i}{ }^{\mu \nu} F^{+}{ }_{\mu \nu} ; F^{+}{ }_{\mu \nu}=\gamma_{i \mu \nu} f^{i},
$$

With this notation, we may express the duals of the Riemann tensor. There are two applications of *:

$$
R_{\alpha \beta \gamma \delta}^{*}=\frac{1}{2} E_{\gamma \delta \sigma \tau} R_{\alpha \beta}^{\sigma \tau} ; * R_{\alpha \beta \gamma \delta}=\frac{1}{2} E_{\alpha \beta \sigma \tau} R_{\gamma \delta}^{\sigma \tau} .
$$

We separate $R_{\alpha \beta \gamma \delta}$ as follows:

$$
R_{\alpha \beta \gamma \delta}=S_{\alpha \beta \gamma \delta}+T_{\alpha \beta \gamma \delta} ; *^{*}{ }_{\alpha \beta \gamma \delta}=S_{\alpha \beta \gamma \delta} ;{ }^{*} T_{\alpha \beta \gamma \delta}^{*}=-T_{\alpha \beta \gamma \delta}
$$

The explicit forms of $S_{\alpha \beta \gamma \delta}$ and $T_{\alpha \beta \gamma \delta}$ are:

$$
\begin{aligned}
& S_{\gamma \delta}^{\alpha \beta}=C_{\gamma \delta}^{\alpha \beta}+\frac{1}{12} R \delta_{\gamma \delta}^{\alpha \beta} ; \\
& T_{\gamma \delta}^{\alpha \beta}=2 R^{[\alpha}{ }_{[\gamma} \delta^{\beta]_{\delta]}} .
\end{aligned}
$$

$C^{\alpha \beta}{ }_{\gamma \delta}$ is the conformal (or Weyl) tensor and is traceless; the traceless Ricci tensor $\dot{R}_{\mu \nu}$ has been defined before. Thus we have

$$
R_{\gamma \delta}^{\alpha \beta}=C_{\gamma \delta}^{\alpha \beta}+2 \AA^{[\alpha}{ }_{[\gamma} \delta^{\beta]}{ }_{\delta]}+\frac{1}{12} R \delta_{\gamma \delta}^{\alpha \beta} .
$$

In $C^{3}, S^{\alpha \beta}{ }_{\gamma \delta}$ and $T^{\alpha \beta}{ }_{\gamma \delta}$ correspond to a symmetric matrix with real trace and a hermitean matrix respectively:

$$
\begin{aligned}
& S_{i j}=\frac{1}{16} \gamma_{i}^{\alpha \beta} \gamma_{j}^{\gamma \delta} S_{\alpha \beta \gamma \delta} ; S=S_{i}^{i}=-\frac{1}{8} R ; \\
& T_{i \bar{j}}=\frac{1}{16} \gamma_{i}^{\alpha \beta} \bar{\gamma}_{j}^{\gamma \delta} T_{\alpha \beta \gamma \delta}=\frac{1}{8}{\gamma_{i}{ }^{\alpha \sigma} \bar{\gamma}_{j \beta \sigma}{ }^{\circ}{ }_{\alpha} .}^{\alpha} .
\end{aligned}
$$

We will also use the notation

$$
\AA_{i j}=S_{i j}-\frac{1}{3} S a_{i j}=\frac{1}{16} \gamma_{i}^{\alpha \beta} \gamma_{j}{ }^{\gamma \delta} C_{\alpha \beta \gamma \delta}
$$

We will use several relations among the $\gamma_{i}{ }^{\alpha \beta}$, including

$$
\begin{aligned}
\gamma_{i}^{\sigma \alpha} \gamma_{j \sigma \beta} & =-a_{i j} \delta_{\beta}^{\alpha}-i E_{i j s} \gamma^{\beta \alpha}{ }_{\beta} ; \\
E_{i j k} & =\sqrt{a} \varepsilon_{i j k} ; a=\operatorname{det}\left(a_{i j}\right) ;
\end{aligned}
$$

${ }^{2}$ An explicit representation of the $\gamma_{i}^{\alpha \beta}$ may be given in a tetrad system in which the metric components are $g_{\alpha \beta}=\eta_{\alpha \beta}$. One such representation, in which $a_{i j}=\delta_{i j}$ is:

$$
\begin{aligned}
& \gamma_{1}{ }^{01}=-\gamma_{1}^{10}=-1, \gamma_{1}{ }^{23}=-\gamma_{1}^{32}=-i \text { all other } \\
& \left.\gamma_{2}{ }^{02}=-\gamma_{2}{ }^{20}=-1, \gamma_{2}{ }^{31}=-\gamma_{2}{ }^{13}=-i\right\} \text { components } \\
& \gamma_{3}{ }^{03}=-\gamma_{3}{ }^{30}=-1, \gamma_{3}^{12}=-\gamma_{3}{ }^{21}=-i \int \text { zero. }
\end{aligned}
$$


which is equivalent to putting an orientation on $C^{3}$. We will also use other easily provable relations such as the symmetry of $\gamma^{i \sigma}{ }_{\alpha} \bar{\gamma}_{j \sigma \beta}$ and $\gamma^{i \sigma \tau} \gamma_{i \alpha \beta}=-\left(\delta_{\alpha \beta}^{\sigma \tau}+E_{\alpha \beta}^{\sigma \tau}\right)$.

The case of a perfect fluid results in a relatively simple form for $T_{i \bar{j}}$ :

$$
T_{i \bar{j}}=\frac{1}{8} \gamma_{i}^{\alpha \sigma} \bar{\gamma}_{j \beta \sigma}(w+p) u^{\beta} u_{\alpha},
$$

where $w$ is the energy density and $p$ the pressure. This $T_{i j}$ is nondegenerate as is seen in a comoving orthonormal tetrad with the expressions of Footnote 8, where $T_{i \bar{j}}=\frac{1}{8}(w+p) \delta_{i j}$.

\section{Discontinuities in the Riemann Tensor}

We now return to the study of discontinuities across a hypersurface $N$. As in Section II, we choose coordinates so that $\left[n_{\mu}\right]=\left[g_{\mu \nu}\right]=\left[g_{\mu \nu, \alpha}\right]$ $=0$; also the $\gamma_{i \mu \nu}$ are continuous. The Riemann tensor may have a nonzero jump across $N$, but the discontinuity in the Riemann tensor is limited because of Eq. (2.1). Eq. (2.1) directly implies that

$$
\left[R^{* \alpha \beta \gamma \delta}\right] n_{\delta}=0 \text {. }
$$

Moreover, the converse theorem is true: If Eq. (4.1) holds, then there is an $\bar{H}_{\alpha \beta}$ such that $\left[R_{\alpha \beta \gamma \delta}\right]$ may be expressed as in Eq. (2.1). The proof is as follows: Eq. (4.1) implies that

$$
\left[R^{* \alpha \beta \gamma \delta}\right]=-E^{\gamma \delta \sigma \tau} A^{\alpha \beta}{ }_{\sigma} n_{\tau}
$$

for some $A^{\alpha \beta}{ }_{\sigma}$. This expression and the symmetry of $* R^{*}{ }_{\alpha \beta \gamma \delta}$ implies, again using Eq. (4.1),

$$
\left[* R^{* \alpha \beta \gamma \delta}\right]=-E^{\alpha \beta \mu \nu} E^{\gamma \delta \sigma \tau} A_{\mu \sigma} n_{\nu} n_{\tau} .
$$

This form for $\left[{ }^{*} R^{* \alpha \beta \gamma \delta}\right]$ readily implies Eq. (2.1). (The result that Eq. (4.1) implies Eq. (2.1) holds whether $n_{\alpha}$ is null or not.)

Note that we have avoided the use of the Bianchi identities

$$
R^{* \alpha \beta \gamma \sigma} ; \sigma=0
$$

in obtaining Eq. (4.1). If Eq. (4.2) is interpreted in the sense of distributions, Eq. (4.1) may be derived by integral methods. We have not made this assumption, however, feeling that the logical procedure is the opposite: Derive Eq. (4.1) from the assumption of admissible coordinates ${ }^{3}$ and then interpret Eq. (4.2) as saying that there are no surface masses on $N$.

${ }^{3}$ Jump conditions have been derived by use of admissible coordinates by, for example [7]. 

form

We return to the study of Eq. (4.1) and write it in the self-left-dual

$$
\left(\left[* R^{* \alpha \beta \gamma \delta}\right]+\left[R^{* \alpha \beta \gamma \delta}\right]\right) n_{\delta}=0 .
$$

The $\gamma_{i}^{\alpha \beta}$ are applied to yield

$$
\left[\stackrel{\Re}{S}_{i j}\right] n_{\delta} \gamma^{j \delta \alpha}+\frac{1}{3}[S] n_{\delta} \gamma_{i}^{\delta \alpha}=\left[T_{i \bar{j}}\right] n_{\delta} \bar{\gamma}^{j \delta \alpha} .
$$

So far we have not required that $n_{\alpha}$ be non-null. We now make this stipulation, and we will retain this requirement through the rest of this paper, unless otherwise stated.

Multiply Eq. (4.3) by $\gamma_{k \beta \alpha} n^{\beta}$ to yield, with the use of Eq. (3.2):

$$
\left[\AA_{i k}\right]+\frac{1}{3}[S] a_{i k}=-\left[T_{i \bar{j}}\right]\left(n^{\sigma} n_{\sigma}\right)^{-1} \bar{\gamma}^{j \delta \alpha} \gamma_{k \beta \alpha} n^{\beta} n_{\delta} .
$$

Eq. (4.4) contains all the information in Eq. (4.3) as may be seen by substitution. Eq. (4.4) is the basic equation which gives the relationships among the discontinuities of the algebraic parts of the Riemann tensor.

$\mathrm{Eq}$ (4.4) does show that the jump in the conformal tensor (represented by $\left[\xi_{i j}\right]$ ) is given by the jump in the stress-energy tensor (which determines $[S]$ and $\left[T_{i \bar{j}}\right]$ ) as was indicated by Eq. (2.3). In fact, the jump in $S_{i j}$, including the jump in $S$ itself, is given by $\left[\dot{R}_{\mu \nu}\right]$.

However, the stress-energy tensor itself cannot have an arbitrary discontinuity across $N$. The analog of Eq. (2.4) in $C^{3}$ notation is

$$
\gamma^{i \sigma \beta} \bar{\gamma}_{\sigma \delta}^{j}\left[T_{i \bar{j}}\right] n_{\beta}+[S] n_{\delta}=0
$$

when the fact that $T_{i \bar{j}}$ is hermitean is used. This equation separates into two parts. The first allows $[S]$ to be determined from $\left[T_{i \bar{j}}\right]$ :

$$
[S]=-\left(n^{\sigma} n_{\sigma}\right)^{-1} \gamma^{i \sigma \beta} \bar{\gamma}_{\sigma \delta}^{j}\left[T_{i \bar{j}}\right] n_{\beta} n^{\delta} .
$$

The second part is three conditions on $\left[T_{i \bar{j}}\right]$ :

$$
\gamma^{i \sigma \beta} \bar{\gamma}_{\sigma \delta}^{j}\left[T_{i \bar{j}}\right] n_{\beta} H_{\alpha}^{\delta}=0, H_{\alpha}^{\delta} \equiv \delta_{\alpha}^{\delta}-\left(n^{\sigma} n_{\sigma}\right)^{-1} n^{\delta} n_{\alpha} .
$$

Eq. (4.7) may be derived directly from Eq. (4.4) when it is noticed that the left side is symmetric in $(i k)$. The condition that the right side also be symmetric is exactly equivalent to Eq. (4.7).

In summary, it has been shown that the jump in the Riemann tensor across a non-null hypersurface $N$ is determined once the jump in $\stackrel{\circ}{R}_{\mu \nu}$ is known. Moreover, any $3 \times 3$ hermitean matrix function on $N, P_{i \bar{j}}$, may represent a jump in $\AA_{\mu \nu}$ across $N$ (that is, $P_{i \bar{j}}=\left[T_{i \bar{j}}\right]$ ) provided $\mathrm{Eq}$. (4.7) is satisfied. Of course Eq. (2.4) is very useful itself, rather than computing $[R]$ from Eq. (4.6).

As a side comment we mention a useful expression for $\left[\dot{S}_{i j}\right]$ in terms of the jump in $T^{\mu \nu}$ and the jump in the scalar curvature $R$ :

$$
\left[\AA_{i k}\right]=-\frac{1}{12}[R] a_{i k}+\frac{1}{4}\left(n^{\sigma} n_{\sigma}\right)^{-1} \gamma_{i}^{\alpha \tau} \gamma_{k \beta \sigma} n^{\sigma} n_{\tau}\left[T_{\alpha}^{\beta}\right] \text {. }
$$


One direct consequence of Eq. (4.4) is a restriction on the algebraic properties of $\left[S_{i j}\right]$. The restriction

$$
\left[S_{j}^{i}\right]\left[S_{k j}^{j}\right]=\left[T^{i}{ }_{\bar{j}}\right]\left[\bar{T}^{j}{ }_{\bar{k}}\right]
$$

arises because $T_{i \bar{j}}$ is hermitean. This fairly simple result will help illustrate the results of the next section.

We may also derive an equation which is the converse of Eq. (4.4). Instead of expressing $\left[S_{i j}\right]$ in terms of $\left[\bar{T}_{i \bar{j}}\right]$ as we did in Eq. (4.4), we express $\left[T_{i j}\right]$ in terms of $\left[S_{i j}\right]$. To do so multiply Eq. (4.3) by $\bar{\gamma}_{k \beta \alpha} n^{\beta}$ and use the complex conjugate of Eq. (3.2) to yield an equation which, like Eq. (4.4), is equivalent to Eq. (4.3):

$$
\left[T_{i \bar{j}}\right]=-\left(n^{\sigma} n_{\sigma}\right)^{-1}\left[S_{i k}\right] n_{\delta} \gamma^{k \delta \alpha} \bar{\gamma}_{j \beta \alpha} n^{\beta}
$$

However, just as the symmetry of $S_{i j}$ is equivalent to the restriction Eq. (4.7) on $\left[T_{i \bar{j}}\right]$, so the hermitean property of $\left[T_{i j}\right]$ results in a restriction on $\left[\xi_{i j}\right]$. The fact that $T_{i \bar{j}}$ is hermitean may be used to show that

$$
\left[\AA_{i k}\right] \gamma^{i \sigma \mu} n_{\sigma} \gamma^{k \delta}{ }_{\nu} n_{\delta}=\left[\bar{\AA}_{i k}\right] \bar{\gamma}^{i \sigma \mu} n_{\sigma} \bar{\gamma}^{k \delta v} n_{\delta} \text {. }
$$

When the tensor $\AA_{i j}$ is interpreted as a conformal tensor, this result becomes

$$
\left[C^{* \sigma \mu \tau \nu}\right] n_{\sigma} n_{\tau}=0 \text {. }
$$

Eq. (4.11) holds whether $n_{\alpha}$ is non-null or null, as can be verified directly from Eq. (2.1).

Thus, we have two alternative ways of looking at jump conditions across $N$. The first starts with a jump in $T_{i \bar{j}}$ which satisfies certain conditions and uses Eq. (4.4) to compute the change in the conformal tensor and scalar curvature. The second specifies the conformal tensor and scalar curvature jumps and computes the change in the traceless Ricci tensor.

The two viewpoints actually use similar "amounts" of information. In the first viewpoint, the nine components of $\left[\AA_{\mu_{\nu}}\right]$ must satisfy the three conditions of Eq. (4.7) (three conditions because $f_{\sigma} H^{\sigma}{ }_{\alpha}$ is automatically perpendicular to $n^{\alpha}$ ). The six remaining functions on $N$ give the full information about changes in the Riemann tensor across $N$. In the second viewpoint, the ten components of $\left[C_{\alpha \beta \gamma \delta}\right]$ are to be given. Since the left side of Eq. (4.11) is automatically orthogonal to $n_{\mu}$ and is tracefree, five conditions result. The five functions left plus the single function representing $[R]$ form the six necessary pieces of information. In Section II we showed that a symmetric tensor $\bar{H}_{\beta \delta}$, orthogonal to $n^{\beta}$, determines the jump in $R_{\alpha \beta \gamma \delta}$. Such an $\bar{H}_{\beta \delta}$ has six independent components, equivalent to the six pieces of information in either viewpoint one or viewpoint two of this section. 
To conclude this section, we will mention to what extent $\left[\dot{S}_{i j}\right]$ is determined in case $N$ is null. In that event, Eq. (4.4) and (4.10) are inappropriate. Instead, these same two equations become conditions on $\left[S_{i j}\right]$ and $\left[T_{i j}\right]$ :

$$
\left[T_{i \bar{j}}\right] \bar{\gamma}^{j \delta \alpha} \gamma_{k \beta \alpha} n^{\beta} n_{\delta}=0 ; \quad\left[S_{i j}\right] \gamma^{j \delta \alpha} \bar{\gamma}_{k \beta \alpha} n^{\beta} n_{\delta}=0
$$

These equations imply that

$$
\left[T_{i \bar{j}}\right] f^{i}=\left[S_{i j}\right] f^{i}=0 ; \quad f_{i}=\gamma_{i}^{\alpha \beta} n_{\alpha} t_{\beta}, \text { with } t_{\sigma} n^{\sigma}=0 ;
$$

$f_{i}$ being a unique null 3-direction. Eq. (4.3) then determines $\left[\AA_{i j}\right]$ (if $[S]$ and $\left[T_{i \bar{j}}\right]$ are given) up to an additive null tensor of the form $A f_{i} f_{j}$. Alternatively, Eq. (4.3) determines $\left[T_{i \bar{j}}\right]$ (if $\left[S_{i j}\right]$ is given) up to an additive term of the form $B f_{i} \bar{f}_{j}$.

\section{Examples: Discontinuities in Fluid-Filled Models}

To illustrate the above remarks, let us compute the algebraic character of the jump in the conformal tensor across a hypersurface of discontinuity in a fluid-filled model. Before treating the most general case, we first look at the situation when $N$ separates a fluid-filled region from a vacuum region:

$$
\left[T_{\mu \nu}\right]=-T_{\mu \nu}^{-}=-\left(w^{-}+p^{-}\right) u^{-}{ }_{\mu} u^{-}{ }_{\nu}-p^{-} g_{\mu \nu} .
$$

As usual we assume that coordinates have been chosen so that

$$
\left[g_{\mu v}\right]=\left[g_{\mu \nu, \alpha}\right]=\left[n_{\alpha}\right]=0 \text {. }
$$

We apply the condition of Eq. (2.4) to find, assuming $w^{-} \neq 0$,

$$
V^{-} \equiv n^{\sigma} u_{\sigma}^{-}=0 ; \quad p^{-}=0 \text {. }
$$

These are the only conditions which need be satisfied when a fluid bounds a vacuum. We may derive Eqs. (5.1) from the $C^{3}$ Eq. (4.7) also. When the jump in $R$ computed from Eq. (4.6) is interpreted as [w-3p], and if $[w]$ is assumed non-zero, we again obtain that $V^{-}=p^{-}=0$.

Once the necessary conditions have been satisfied we compute the jump in the conformal tensor by using Eq. (4.4). Since $V^{-}=0, n_{\alpha}$ is spacelike, and we may normalize it: $n^{\sigma} n_{\sigma}=+1$. Eq. (4.8) yields the result:

$$
\left[\AA_{i k}\right]=\frac{1}{12} w^{-} a_{i k}-\frac{1}{4} w^{-} X^{-}{ }_{i} X_{-}^{-} ; \quad X^{-}{ }_{i} \equiv \gamma_{i}^{\alpha \sigma} u^{-}{ }_{\alpha} n_{\sigma}
$$

Because $X^{-}{ }_{i}$ is unit, $X^{-i} X^{-}{ }_{i}=X^{-}{ }_{i} X^{-}{ }_{j} a^{i j}=1$, we see that $\left[\dot{S}_{i k}\right]$ is indeed traceless.

Eq. (5.2) shows that in the case of a fluid bordering on a vacuum the jump in $\$_{i j}$ is algebraically of type D. This type of conformal tensor is characterized by the fact that all type D tensors $\AA_{i j}$ satisfy a second 
order equation of the form

$$
\left(\dot{S}^{i}{ }_{j}-\lambda \delta^{i}{ }_{j}\right)\left(\dot{S}^{i j}{ }_{k}+2 \lambda \delta^{j}{ }_{k}\right)=0 .
$$

The $\left[\AA_{i j}\right]$ of Eq. (5.2) does satisfy a second order equation of this form, with the eigenvalue $\lambda$ given by

$$
\lambda=\frac{1}{12} w^{-} \text {. }
$$

We could have used Eq. (4.9) directly to discover the algebraic character of $\left[\hat{S}_{i j}\right]$. In our case, the product $\left[T_{\bar{j}}^{i}\right]\left[\bar{T}_{\bar{k}}\right]$ is simply proportional to $\delta^{i}{ }_{k}$. Eq. (4.9) then becomes the second order equation which $\left[\AA^{i}{ }_{j}\right]$ satisfies.

A familiar example of this type of discontinuity is that between the Schwarzschild interior and exterior solutions. The Schwarzschild interior solution is a conformally flat $\left(C_{\alpha \beta \gamma \delta}=0\right)$, static, fluid model. The Schwarzschild vacuum model, which surrounds the interior solution, is type $\mathrm{D}$ not only on the fluid boundary but also everywhere else.

We now turn to the case of a hypersurface of discontinuity lying within a fluid. The jump in the stress-energy tensor is

$$
\left[T_{\mu \nu}\right]=\left(w^{+}+p^{+}\right) u^{+}{ }_{\mu} u^{+}{ }_{\nu}+p^{+} g_{\mu \nu}-\left(w^{-}+p^{-}\right) u^{-}{ }_{\mu} u^{-}{ }_{\nu}-p^{-} g_{\mu \nu} .
$$

To the jump condition, Eq. (2.4), must be appended two additional conditions in order that the jump in fluid parameters be determined. The first of these conditions is

$$
\left[\varrho u^{\mu}\right] n_{\mu}=0
$$

which expresses the conservation of particle number density $\varrho$. In interpreting Eq. (5.3) and (2.4), the pressure $p$ must be related to $\varrho$ and the specific entropy $S$ by the caloric equation of state $p=p(\varrho, S)$. The second condition is that if fluid crosses the discontinuity, then $S$ must not decrease [8].

There are two classes of fluid discontinuities, Class I, in which there is no transfer of matter across $N$, and Class II, in which there is such transfer.

We define

so that Eq. (2.4) is

$$
V^{+} \equiv u^{+}{ }_{\mu} n^{\mu} ; \quad V^{-} \equiv u^{-}{ }_{\mu} n^{\mu}
$$

$$
\left(w^{+}+p^{+}\right) u^{+}{ }_{\mu} V^{+}-\left(w^{-}+p^{-}\right) u^{-}{ }_{\mu} V^{-}+[p] n_{\mu}=0 .
$$

The first class of discontinuities occurs when $\varrho^{+} V^{+}=\varrho^{-} V^{-}=0$. If $\varrho^{+}$ and $\varrho^{-}$are both non-zero, then $V^{+}=V^{-}=0$. Eq. (5.4) then shows that

$$
[p]=V^{+}=V^{-}=0 \text {. }
$$

Eq. (5.3) shows that in the only other class of discontinuities $V^{+}$and $V^{-}$ are both non-zero. (In the case of a fluid-vacuum jump, Class I, one of the $V$ 's is undefined.) 
For Class II discontinuities ("shocks") Eqs. (5.3) and (2.4) along with the caloric equation of state determine the fluid parameters on one side of $N$, say the "+" side, in terms of the fluid parameters on the "-" side as functions of one parameter. This parameter may be taken as the shock velocity, related to $V^{-}$, or the shock strength, say $[\varrho] / \varrho^{-}$. Thus, for example, one finds the Hugoniot curve relating those $(p, \varrho)$ states which can be obtained from a fixed $\left(p^{-}, \varrho^{-}\right)$by passing through a shock. The condition that $S$ not decrease as the fluid passes through the shock then eliminates the possibility of negative shocks [9].

The discussion of the thermodynamic properties of fluids is outside the scope of this paper, and we will merely assume that the fluid parameters are known on both sides of the shock front.

Class II can occur only when ${ }^{4}$

$$
C^{2} \equiv\left(u^{+}{ }_{\sigma} u^{-\sigma}\right)^{2}-1=\frac{[w][p]}{\left(w^{+}+p^{+}\right)\left(w^{-}+p^{-}\right)} .
$$

Eq. (5.5) is readily derived from Eq. (2.4) and the condition $V^{+}$and $V^{-}$ both non-zero. Contract Eq. (5.4) with $u^{-\mu}$ and again with $u^{+\mu}$ and $n^{\mu}$. In case Eq. (5.5) holds, the resulting equations may be used to show

$$
\left(V^{-}\right)^{2} \frac{\left(w^{-}+p^{-}\right)}{\left(w^{+}+p^{-}\right)}([w]-[p])=n_{\sigma} n^{\sigma}[p],
$$

which is similar to the Eq. (6.8) given by TAUB [9]. Because the shock wave must travel slower than light, $n_{\sigma}$ is spacelike and may be normalized:

$$
n_{\sigma} n^{\sigma}=+1 \text {. }
$$

We may then find $V^{-}$and $V^{+}$in terms of $w^{+}, w^{-}, p^{+}$, and $p^{-}$. The following relation then holds: $u^{+}{ }_{\mu}$ is uniquely given in terms of $u^{-}{ }_{\mu}$ and $n_{\mu}$ if $w^{+}, w^{-}, p^{+}, p^{-}$are known. Explicitly, we find

$u^{+}{ }_{\mu}=\left\{\frac{\left(w^{-}+p^{-}\right)\left(w^{+}+p^{-}\right)}{\left(w^{+}+p^{+}\right)\left(w^{-}+p^{+}\right)}\right\}^{1 / 2} u^{-}{ }_{\mu}-\left\{\frac{[p]([w]-[p])}{\left(w^{+}+p^{+}\right)\left(w^{-}+p^{+}\right)}\right\}^{1 / 2} n_{\mu}$.

It may be readily verified that if $w^{+}$and $p^{+}$satisfy the relation

$$
u^{-}{ }_{\sigma} n^{\sigma}=V^{-}=\left\{\frac{\left(w^{+}+p^{-}\right)[p]}{\left(w^{-}+p^{-}\right)([w]-[p])}\right\}^{1 / 2},
$$

then $\left[T^{\mu \nu}\right] n_{\nu}$ does vanish, provided $u^{+}{ }_{\mu}$ is determined as in Eq. (5.6). (We have presumed $[p]>0$.)

The computation of $\left[\AA_{i j}\right]$ for discontinuities of Class I with $\varrho^{+}$and $\varrho^{-}$non-zero is straightforward. Because $p^{+}=p^{-}=p$ and because $V^{+}=V^{-}=0,\left[T^{\mu \nu}\right]$ is simply a difference of two terms similar to the $\left[T^{\mu \nu}\right]$ which occurs when a fluid borders a vacuum. The jump in $\AA_{i j}$ for a Class I internal discontinuity is therefore the difference of two terms

4 The proof that two forward-pointing timelike unit vectors, $u^{+\mu}$ and $u^{-\mu}$, have a dot product $u^{+\mu} u^{-}{ }_{\mu}$ less than or equal to -1 is straightforward. 
of the form of Eq. (5.2). We thus have:

where

$$
\begin{gathered}
\text { Class I: }\left[\AA_{i j}\right]=-\frac{1}{12}[w] a_{i j} \\
+\frac{1}{4}\left(w^{+}+p\right) X^{+}{ }_{i} X^{+}{ }_{j}-\frac{1}{4}\left(w^{-}+p\right) X^{-}{ }_{i} X^{-}{ }_{j},
\end{gathered}
$$

$$
X_{i}^{ \pm} \equiv \gamma_{i}^{\mu v} u^{ \pm}{ }_{\mu} n_{\nu}
$$

The $X^{ \pm}{ }_{i}$ are unit but not orthogonal:

$$
X^{+}{ }_{i} X^{+i}=X^{-}{ }_{i} X^{-i}=1 ; \quad X^{+}{ }_{i} X^{-i}=-u^{+}{ }_{\sigma} u^{-\sigma}=\left(1+C^{2}\right)^{1 / 2} .
$$

(Of course, in Class I, $C^{2}$ does not obey Eq. (5.5).)

The algebraic class of $\left[\AA_{i j}\right]$ is type I except for two of the three special cases defined below. Tensors $\AA_{i j}$ of type I satisfy a third order equation

$$
\begin{gathered}
\left(\AA^{i}{ }_{j}-\lambda_{1} \delta^{i}\right)\left(\AA^{j}{ }_{k}-\lambda_{2} \delta_{k}^{j}\right)\left(\aleph^{i k}{ }_{m}-\lambda_{3} \delta^{k}{ }_{m}\right)=0 ; \\
\lambda_{1}+\lambda_{2}+\lambda_{3}=0 ; \quad \lambda_{1} \neq \lambda_{2} \neq \lambda_{3} \neq \lambda_{1}
\end{gathered}
$$

but no second order equation. It may be readily verified that the three eigenvalues of the $\left[\dot{S}_{i k}\right]$ are

$$
\begin{aligned}
& \lambda_{1}=-\frac{1}{12}[w] \\
& \lambda_{2}=\frac{1}{24}[w]+\frac{1}{8}\left\{[w]^{2}-4\left(w^{+}+p\right)\left(w^{-}+p\right) C^{2}\right\}^{1 / 2} \\
& \lambda_{3}=\frac{1}{24}[w]-\frac{1}{8}\left\{[w]^{2}-4\left(w^{+}+p\right)\left(w^{-}+p\right) C^{2}\right\}^{1 / 2} .
\end{aligned}
$$

There are three special cases of Class I discontinuities, which will be mentioned here, but not elaborated on. First is the subclass $u^{+}{ }_{\mu}=u^{-}{ }_{\mu}$, so that $C=0$. In this special subclass we may include the case $w^{+}=p=0$ even though properly speaking $u^{+}{ }_{\mu}$ is undefined in the fluid-borderingvacuum case. In this subclass there are only two unequal eigenvalues. $\left[\dot{S}_{i j}\right]$ is Type D and obeys a second-order equation. (The fluid-vacuum case was treated above; the fact that the conformal jump in the more general case of $u^{+}{ }_{\mu}=u^{-}$is Type D follows from Eq. (5.7).)

The second special case occurs when $w^{+}, w^{-}, p$ satisfy the relation

$$
[w]^{2}=4\left(w^{+}+p\right)\left(w^{-}+p\right) C^{2} \text { with } C \neq 0 \text {. }
$$

This case corresponds to the limit $\lambda_{2} \rightarrow \lambda_{3}$, but unlike the Type I case is not diagonalizable. In this special case $\left[\AA_{i j}\right]$ obeys a third-order equation with two equal eigenvalues and does not obey any second-order equation. This fact may be seen by direct computation using Eq. (5.7). This behavior characterizes a tensor of algebraic Type $I I I$.

The final case of interest occurs when $\lambda_{3}$ vanishes so that

$$
2[w]^{2}=9\left(w^{+}+p\right)\left(w^{-}+p\right) C^{2} .
$$


This third case is type $I$ and corresponds to an $\left[\hat{S}_{i k}\right]$ which is the difference of two type $N$ tensors.

We now turn to the Class II discontinuities, which correspond to shock waves moving through fluids. The equations concerning the physical properties of the fluid itself have been well discussed in the literature $[1,2,9]$. Here we are concerned with the algebraic form of the jump in the conformal tensor associated with such a shock front.

Because $u^{+}{ }_{\mu}$ is related to $u^{-}{ }_{\mu}$ by Eq. (5.6), the expression for $\left[\AA_{i k}\right]$, given by Eq. (4.8), becomes

We have set

$$
\left[\AA_{i k}\right]=-\frac{1}{12}[w] a_{i k}+\frac{1}{4}[w] Y_{i} Y_{k} .
$$

$$
Y_{i}=\left[1+\left(V^{+}\right)^{2}\right]^{-1 / 2} \gamma_{i}{ }^{\alpha \beta} u^{+}{ }_{\alpha} n_{\beta}=\left[1+\left(V^{-}\right)^{2}\right]^{-1 / 2} \gamma_{i}{ }^{\alpha \beta} u^{-}{ }_{\alpha} n_{\beta},
$$

so that $Y_{i}$ is unit: $Y_{i} Y^{i}=1$. Therefore, in all Class II cases $\left[\AA_{i k}\right]$ is type $D$, and the eigenvalues of $\left[\dot{S}_{i k}\right]$ depend only on the discontinuity in the energy density $w$ and not explicitly on the discontinuity in the pressure $p .^{\mathbf{5}}$

\section{Summary and Conclusion}

We have explored the conditions that the assumption of admissible coordinates places on second-derivative discontinuities across a non-null hypersurface $N$ in general relativity. We showed that the jump in the traceless Ricci tensor $\stackrel{R}{\mu \nu}_{\nu}$ may be arbitrarily specified except for the three conditions represented by Eq. (4.7). The discontinuity in the full Riemann tensor is then uniquely determined from $\left[\mathscr{R}_{\mu \nu}\right]$ by Eq. (4.4).

A physically more interesting procedure is to specify $\left[T^{\mu \nu}\right]$ subject to the conditions

$$
\left[T^{\mu \nu}\right] n_{v}=0 .
$$

If $T^{\mu v}$ is required to be of a fluid form on both sides of $N$, Eq. (6.1) must be supplemented by Eq. (5.3) and the caloric equation of state. $N$ is taken as timelike $\left(n_{\mu}\right.$ spacelike) for reasons of causality, and the specific entropy $S$ is required to be non-decreasing by the second law of thermodynamics. The fluid parameters on one side of $N$ are then related to those on the other side in terms of the shock strength parameter.

Once a $\left[T^{\mu \nu}\right]$ and a hypersurface $N$ which are mutually compatible (in that Eq. (6.1) is satisfied) are found, the conformal tensor jump is then uniquely determined. If the conformal tensor does represent in

5 According to a student of his, R. Boyer did some work involving computation of conformal tensor discontinuities across fluid boundaries. R. WILson tells me that BOYER proved that the conformal tensor jump across a fluid-vacuum boundary is type $\mathrm{D}$ and that the conformal tensor jump across an internal null hypersurface in a fluid is type $D$ plus an admixture of type $N$. This latter situation can occur only when $[p]=[w]$. 
some sense the "purely gravitational" part of the field, then it is reasonable to expect that it is determined by the matter variables, at least to this extent.

For discontinuities involving fluid stress-energy tensors, the conditions imposed are well known [1, 2,9]. The allowable discontinuities are of two classes: I, in which no matter crosses $N$, and II, in which there is matter transfer. We have here computed the jump in the conformal tensor associated with both classes of jumps.

For Class I boundaries (represented by the boundary between a layer of oil floating on a layer of water) the jump in $C^{\alpha \beta}{ }_{\gamma \delta}$ is of type $I$, nondegenerate, except for special cases. For external boundaries, in which the fluid borders on a vacuum, the algebraic type of $\left[C^{\alpha \beta}{ }_{\gamma \delta}\right]$ is Type $D$, however. For Class II discontinuities (represented by a shock wave moving through a fluid) the jump in the conformal tensor is always of algebraic Type $D$.

In conclusion, two points should be raised. The first is that the jump conditions deal only with the algebraic properties of $T^{\mu \nu}$ and $C^{\alpha \beta}{ }_{\gamma \delta}$ on the hypersurface $N$. When it is required that $T^{\mu \nu}$ or $C^{\alpha \beta}{ }_{\gamma \delta}$ obey an algebraic restriction in an open neighborhood of $N$, further jump conditions must be imposed. This subject will be treated in a subsequent paper.

The second point is that singular hypersurfaces of infinite density do find use within general relativity. Since the use of admissible coordinates results in the jump conditions (4.1), the Bianchi identities Eq. (4.2) may then be interpreted as indicating that $N$ has no "surface charge". Conversely, if a surface of point masses is to be described, admissible coordinates must be dispensed with. In that case the jump conditions should be derived by approximating the surface layer by a thick sheet of mass whose thickness approaches zero. The Bianchi identities (4.2) could in this case be the starting point for the derivation of jump conditions.

Acknowledgement. The author wishes to thank Professor A. H. TAUB of the University of California at Berkeley for his advice and helpful criticism.

\section{References}

1. O'Brien, S., and J. L. Synge: Comm. Dublin Inst. Adv. Stud. A 9 (1952). Lrohnerowicz, A.: Theories Relativistes de la Gravitation et de l'Electromagnetism. Paris: Masson 1955.

TAUB, A. H.: Illinois J. Math. 1, 370 (1957).

2. See, for example, LICHNERowICz, A.: Relativistic hydrodynamics and magnetohydrodynamics. New York: W. A. Benjamin 1967.

3. Cocke, W. J.: J. Math. Phys. 7, 1171 (1966).

4. Estabrook, F. B., and H. D. Wahlquist: J. Math. Phys. 8, 2302 (1967). 
5. BeL, L.: Compt. Rend. Acad. Sci. Paris 245, 2482 (1957).

6. An easily accessible reference is: $\mathrm{T}_{A U B}, \mathrm{~A}$. H.: In: Perspectives in geometry and relativity. (Ed. by B. HofFmann.) p. 360. Indiana Univ. 1966.

7. ScHILD, A.: In: Lectures in applied mathematics. Relativity theory and astrophysics. Am. Math. Soc. 8, 1 (1967). Also see BeL, L., and A. Hamour: Les Conditions de Raccordement. Preprint.

8. Courant, R., and K. O. Friedrichs: Supersonic flow and shock waves. New York: Interscience 1948.

9. TAUB, A. H.: In: Lectures in applied mathematics. Relativity theory and astrophysics. Am. Math. Soc. 8, 170 (1967).

\section{C. SHePLeY}

The University of Texas

Department of Physics

Austin, Texas 78712

USA 